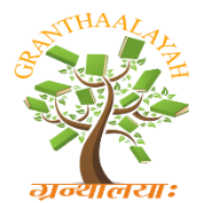

\author{
INTERNATIONAL JOURNAL OF RESEARCH - \\ GRANTHAALAYAH \\ A knowledge Repository
}

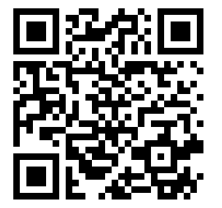

Science

\title{
NUTRITIONAL HEALTH STATUS WITH DIFFERENT ASPECT OF TARGET GROUP (PREGNANT AND LACTATING WOMEN): A LITERATURE REVIEW
}

\author{
Rajeev Singh Dhakad ${ }^{* 1}$, Abhishek Kumar ${ }^{2}$ \\ ${ }^{*}$ State Co-Ordinator, Indian Institute of Health Management Research, Jaipur, Rajasthan, India \\ ${ }^{2}$ Analyst, MIHM, Indian School of Business, Mohali, Punjab, India
}

\begin{abstract}
The pregnant women and lactating women are considered as the nutritionally exposed group specifically in the emerging countries. The present paper is mainly focusing on nutritional intake status/behaviours with respect to the culture, beliefs, habits, socioeconomic status and the knowledge and awareness of the target group i.e. pregnant and lactating women.

Method: we were review various literature and studies with the keywords such as nutrition, dietary intake, food intake behaviour, culture and practices. In this paper literature were divided it into three sections. These were (a) Nutrient and Dietary Intake; (b) Cultural Beliefs and Practices; (c) Socio-Economic Characteristics.

Results: Nutrient and dietary intake were not matched with recommended RDA. Cultures beliefs and practices were very changed in the different community and economic groups such as tribal community were not taking important food during pregnancy and lactation period and Urban women had the higher knowledge of nutrition diet compared to rural women. Socio-economic condition influences the dietary behavior or food's choice of pregnant and lactating women.

Conclusions: Various studies and papers were reviews in this paper, we found gaps between recommended RDA, nutritional behavior, and practices in the target groups of developing countries such as India. This paper concludes the further research and intervention are required on pregnant and lactating women on nutritional behaviour as well as dietary practices.
\end{abstract}

Keywords: Nutritional Health; Dietary Intake; Nutritional Behaviour; Culture, Beliefs.

Cite This Article: Rajeev Singh Dhakad, and Abhishek Kumar. (2019). "NUTRITIONAL HEALTH STATUS WITH DIFFERENT ASPECT OF TARGET GROUP (PREGNANT AND LACTATING WOMEN): A LITERATURE REVIEW." International Journal of Research Granthaalayah, 7(5), 38-48. https://doi.org/10.29121/granthaalayah.v7.i5.2019.822.

\section{Introduction}

A complete review of the literature is vital for all research because it delivers an up-to-date understanding of the subject and its implication to research and classifies the procedures used in previous research on the topic which further helps us in delivering the objectives. 
The available references, supportive or contradictory, also help in drawing conclusions during the research process. Although many studies have been made on nutrition, nutritional status and nutritional intake of pregnant and lactating women, only a few researches are available on food intake pattern or dietary advice for pregnant and lactating among the study group. The comprehensive search did not produce even any significant review relating to the development of any evidence-based and actionable dietary advice for pregnant and lactating women and assessment of its impact on pregnant and lactating women based on the 24-hour recall method.

The pregnant women and lactating mothers are thought as the vulnerable group specifically in the emerging countries. The requirement of nutrient in a body has increased at the time of pregnancy and lactation period therefore that Proper nutrient is very essential during pregnancy and lactation period. Foods consumed by many pregnant women and lactating mothers in our country are poor and lack in many nutrients. Several Studies showed that the Nutrient and dietary intake by the pregnant and lactating women is very low in comparison to Recommended Dietary Allowances (RDA).

Many researchers have done study to define the nutritional status of pregnant and lactating women in India. This paper is going to include numerous researches into the food and nutrient intake as well as effects of socio-economic characteristics and cultural beliefs \& practices on dietary intake by pregnant and lactating women in India.

An optimal level of nutrition in the diet is the most fundamental criteria for ensuring good maternal and child health especially during the pregnancy and lactation period. In the lactation period the nutritional and health stage of the new born child depends on the mother. Effective pregnancy and lactation require changes in the body for the function of various physiological systems. So, a diet that meets all the nutritional needs is required for these changes so that the wellbeing of the mother is ensured with the birth of healthy child [1].

The Indian Council of Medical Research expert committee, taking interested in consideration the nutrient necessities, has suggested that since requirements of iron and folic acid are more require for pregnant women they should eat $100 \mathrm{~g}$ of leafy vegetables daily [2].

Pregnancy and lactation are also energy and macronutrient need in lactating women are higher than pregnant women. Likewise, the intake recommendations (RDA) for macronutrients and minerals and vitamins are higher in the case of lactating women compared to the pregnant women and should be met as per the requirements stated in the guidelines (Food and Nutrition Board, 1991).

To get the idea of current situation related to pregnant and lactating women, we went through the various studies with the keywords like nutrition, dietary intake, food intake etc. and divided it into three sections taking the different aspects like socioeconomic status, myths, believes regarding intake etc. 


\section{Objectives}

The present paper was undertaken to review of nutritional intake status/behaviours with respect to the culture, beliefs, habits, socioeconomic status and the knowledge and awareness of the target group i.e. pregnant and the lactating women.

\section{Methodology}

For the review, we included original published articles, articles in press, reports, conference abstracts, conference papers, conducted in India or in other countries based on pregnant and lactating women. The focus was on the most recent studies as of acceleration in the nutrition transition in recent years in India because of the various like the increase in literacy level, health system and the reach of the people.

The initial search identified 57 articles from different databases. These databases are PubMed, google scholar, and open access journals etc. PubMed was the prime database for this paper. The titles and abstracts of all 57 articles were screened, with 35 being excluded as they did not match the inclusion criteria. Twenty-two studies were accessed as full papers. For clarity, the review has been arranged in sections and subsections, the findings have been categorized into 3 broad sections

\section{(I) Nutrient and Dietary Intake; (II) Cultural Beliefs and Practices; (III) Socio Economic Characteristics.}

The first section of this literature review, we have studied the various studies on the dietary intake and nutrient intake by pregnant and lactating women in India. This section summarized the results of several studies about the dietary and nutritional intake of pregnant and lactating women in India. In the second section, we have tried to compile the studies of different authors define the studies on cultural issues during pregnancy and lactating period those have the effect on the nutritional status of women.

The last section of this literature review, light have been thrown on the effects of socioeconomic characteristics on dietary and nutrient intake by the pregnant and lactating women by the different studies. Socioeconomic characteristics have a great influence on the dietary intake by the women so that in this review section the author describes the various studies results for affecting factors to the status of pregnant and lactating women in India in terms of nutrition. The conclusions can be used to strategy a new or modified nutritional intervention programme directing to deliver the nutritional awareness, knowledge, and practices in women to ultimately improve the alimentary health status of the pregnant and lactating women.

\section{Results and Discussions}

\subsection{Nutrient and Dietary Intake}

The available studies show that nutrition outcomes are affected by a range of socio-cultural factors, feeding, and dietary practices and lifestyle factors. These are the important determinants of nutrition and health status of the pregnant and the lactating women of India. 
In one of the community based cross-sectional survey conducted in six villages of a rural area of district Faridabad in Haryana state, India among the pregnant women aged 18 years or more, with pregnancy duration of more than 28 weeks, there was a high occurrence of micronutrient deficits amongst the Pregnant Women of the area, probably due to the poor dietary intake of diet and very low frequency of consumption of food groups which are good for the micronutrients. The coexisting prevalence of more than two or three micronutrient deficiencies were common [3].

In an innovative study, 28 villages in Jodhpur district out of 384 pregnant women, 400 lactating women and 409 non-pregnant non-lactating women (15 years and older, women who have not reached their family status), reported and it was found that the fat and cereals was below the Recommended Dietary Intakes (RDA) of the Indian Council of Medical Research (ICMR). The consumption of legumes and legumes was very low, ranging from 47 to $65 \%$ of the GDR and very low leafy vegetables, or from $12 \%$ to $7 \%$ of the GDR in pregnant and lactating women, but found a milk intake to be adequate.

This study also examined a high protein and energy deficit in pregnant and lactating women (20 to $40 \%$ ) and also showed a micronutrient deficiency (vitamin A) in breastfeeding women. This study evaluated the extent of three disorders due to micronutrient deficiency (iron, vitamin A and iodine), nutritional deficiencies and their association with related factors. The study found that the majority of women were anemic. Anemia was higher in pregnant and lactating women (80.7\%). Severe anemia was three times higher in pregnant and lactating women than in controls (4.1\%). Vitamin A deficiency was higher in pregnant women (8.8\%). A high proportion of women (80.8\%) consumed salt, as their iodine content was inadequate. Median urinary iodine values were lower in pregnant and lactating women compared to WHO thresholds. Pulses and legumes were low along with leafy vegetables. The average nutrient intake showed a deficiency of protein and energy, iron and folic acid and vitamin A deficiency. The study concluded that the proportion of anemia in this study was higher compared to the studies. conducted at national level in addition to the low consumption of normal iodized salt [4].

Bhatia et al. found that the rural and urban breastfeeding mothers' diets in Varanasi were deficient in calories, calcium, retinol, ascorbic acid and niacin compared to ICMR recommendations. this survey was conducted on breastfeeding mothers and it was reported that average dietary intakes covered only $73 \%$ of energy recommendations, $79 \%$ protein, $52 \%$ calcium, $89 \%$ iron and $55 \%$ iron. Vitamin A, 95 lactating vegetarian women and 20 vegetarian vegetarians from urban and rural areas of Varanasi (NPNL) were subjected to an analysis of their dietary intake. The diet of pregnant and lactating women was insufficient in calories, calcium, retinol and riboflavin, contrary to the recommendations of the Indian Council of Medical Research. Pregnant women also absorbed less iron and riboflavin. Urban pregnant women's intakes were lower and those of pregnant women in rural areas higher than those of their counterparts in the NPNL. Mean intakes of various nutrients among lactating women living in urban and rural areas were higher than those of pregnant women and NPNL women. Lactating women living in urban areas outnumbered lactating women living in rural areas. There appeared to be insufficient dietary intake rather than protein deficiency [5]. Another study also found that the consumption of foods, namely cereals, legumes, GLV, roots and tubers, other vegetables, fruits and sugar, with the exception of milk and dairy products at urban mothers and fats and oils in rural nursing mothers was low. The study also 
found that consumption of all foods was lower than recommended, resulting in low nutrient intake [6].

But if we talk about food consumption comparing with the Muslim population, we found that among pregnant women, the Muslim population shows better consumption of all the nutrients (iron, protein, calories, etc.), which could be attributed to its comparison with the non-Muslim population. But she did not mention the gravid status or the size of the family that is primarily responsible for anemia in the Muslim community, according to another study [7].

Studies of women in other states also give a similar result to that of women in Rajasthan. If we talk about the Jood et al study that was conducted on the nutritional status of pregnant women in rural areas in three districts of Haryana State. 90 pregnant women in rural areas were selected for this study. The study found that the diet consisted mainly of cereals, legumes, roots and tubers, other vegetables, sugar and jaggery. iron, thiamine, riboflavin, niacin and ascorbic acid. The consumption of milk and milk products and fats and oils was much higher than that of the RDI, while the consumption of green leafy fruits and vegetables was very low [8]. This can also be seen in the study by Agarwal et al. that energy and protein intakes were lower than the recommended dietary intakes in all selected states in the study. The study also indicated that vitamin A intake was also lower than RDA in all states except those included in the study. Fat consumption was greater than RDA during pregnancy and increased more during lactation. Vitamin $\mathrm{C}$ intake was close to the GDR except for Haryana and Assam [9].

Similarly, Andersen et al., A study, also reports on dietary intake among pregnant women in rural Tamilnadu, southern India. The study found that respondents' intake of cereals and vegetables was significantly lower than that recommended by the Indian. Fruit consumption was higher than recommended, while legumes, green leafy vegetables (GLV), milk, oils and fats, and sugar and Jaggery were the most limited food items. The study indicates that the average consumption of green leafy vegetables was very low and that the fruits were more than three times higher than the recommended consumption. The study defines that cereals provided $57 \%$ of protein intake and $46 \%$ of iron intake. The nutritional intake of women, with the exception of fat, was lower than the recommendations [10].

The done by the very unique methodology of 24-hours recall also compiles the same result. The dietary study conducted by "24-hour recall" method for 1 day indicated that the consumption of all the foods i.e. fruits $(13.33 \%, 35.50 \%)$, roots and tubers $(20.25 \%, 17.66 \%)$, milk and milk products $(23.16 \%, 31.66 \%)$, green leafy vegetables $(43.38 \%, 46.50 \%)$ and pulses $(14.44 \%$, $26.61 \%)$ and, fats and oil $(77.00 \%, 75.26 \%)$ other vegetables $(60.00 \%, 50.14 \%)$ and meat and meat products $(91.77 \%, 93.24 \%)$ except cereals and millets $(86.60 \%, 81.10 \%)$ were below the suggested levels. The mean intake of all nutrients (i.e. protein, calcium, iron, thiamin, niacin fat, energy, vitamin $\mathrm{A}$, riboflavin, vitamin $\mathrm{C}$ and free folic acid) by the lactating women belonging from Saharia tribal community of Baran District, were found to be inadequate [11].

The study claimed to understand the factors influencing the dietary and nutritional intake of pregnant women in an urban settlement in Delhi, India. The study showed that dietary intake of pregnant women was not significantly different from that of their non-pregnant counterparts in terms of food group and nutrient intake. Median consumption of fruit, meat and poultry products 
was zero in both groups. A cereal was the main source of energy intake. The study also found that intake of energy, protein, iron, calcium, vitamin $\mathrm{C}$ and folic acid was low in pregnant women and non-pregnant women compared to the Recommended Daily Allowance (RDA) [12].

Another study of women in the slums of Delhi revealed the same result, namely the eating habits and nutritional status of pregnant and lactating women. The study found that one third of women had insufficient energy intake (PW (36\%), RDW (newly delivered women 34.2\%)). This study also found that the average micronutrient and macronutrient intake was lower than that recommended [13].

A longitudinal study measured the dietary intake of pregnant women in Pune District, Orissa State. The energy intake of breastfeeding women was almost similar to RDI, while that of protein, calcium, phosphorus, carbohydrates, iron and zinc was lower. But there were many other positive things in the study compared to studies done on women in Rajasthan and other states. According to the author, among these relatively sedentary urban women belonging to the average socioeconomic class of western India and consuming high-fat foods, an interesting positive result was found to be a significant $(11 \%)$ increase in the percentage of of android fat. at 6 months remaining high at 1 year. In this study, all women consumed approximately 2 to 3 times more dietary fat than the recommended amounts [14].

The study by Sinha et al., Which also concludes that all tribal pregnant women consume three meals a day, also reveals this type of positive dietary intake. Cereal intake was significantly higher than the recommended dietary intake. Consumption of other vegetables was almost similar to IDR, while that of other foods was well below IDR. The daily intake of calories, protein, calcium, iron and b-carotene was clearly insufficient compared to ICMR recommendations. The study found that one hundred percent of women were not safe in terms of calcium, fat, iron and b-carotene, while only $2 \%$ and $9 \%$ of women were safe with respect to energy and proteins, respectively. But this type of positive results is much lower than other studies, which shows the negative results [15].

\subsection{Cultural Beliefs and Practices}

In India, many cultural beliefs and practices are the basis for the Dietary pattern during pregnancy and lactation. Pregnant and lactating women have restrictive diet during their period, due to their traditional beliefs and practices without any scientific reason. The authors reviewed some studies those defined the restrictive diet during pregnancy and lactation period. Eating nutritionally balanced foods during pregnancy is very essential. In India, dietary habits of pregnant women are highly influenced by food fads, cultural taboos, customs, and religious beliefs. The study also reflects that half of the women among the target group stated that they were increased their dietary intake on the advice of Doctors and family members.

Agrahar-Murugkar and Pal's study of 650 Khasi tribal women over the age of 18 examined dietary habits, beliefs and trends that contribute to women's nutrition and health. The study of energy consumption, protein, iron and vitamin $\mathrm{C}$ was adequate in breastfeeding women (L) in whom the level of energy was significantly lower than the daily intake. Pregnant women in low income group (LIG) and high-income group (HIG). The consumption of pulses, dairy products, other vegetables, 
fats and oils has been considerably reduced, resulting in low consumption of fat, calcium and carotene in all physiological states and groups of fats. income. The study suggests dietary modifications to compensate for dietary deficiency in calcium, fat and carotene, especially during pregnancy and lactation, and the integration of nutrition education into community development programs [16].

Similarly, in one of the evaluation studies of dietary habits and customs of the Bhil tribe of Jhadol and Girwa panchayat samiti of Udaipur district, it was reported that the Bhils' dietary habits are greatly influenced by beliefs, the traditions, customs and taboos of society. All respondents were not vegetarians. They consider the importance of special foods in various physiological conditions, namely pregnancy, breastfeeding, sickness and fasting. Because of their adverse socio-economic conditions, they cannot afford important foods in such conditions. Very few women in Bhil have a special diet during pregnancy and breastfeeding [17].

The studies of said that it's not the only culture of a region but the family system and their customs are also a major factor in taking the decision about the dietary pattern. The study found that the eating customs may be an obstacle to consuming the recommended amounts of foods [10,12]. This studies also reflects restrictive diet by the women during their trimester period influenced by the role of family and customs, women stated that while they were asked to restrict some of the food items during pregnancy, intake of some is also encouraged by their family members. Restricted food items include papaya, egg, and chicken. Egg and chicken were considered as hot food. Papaya was being restricted because of the belief that it can lead to miscarriage $[10,12]$.

Most women have changed their eating habits during pregnancy. They reported consuming the following foods more frequently and / or in larger quantities such as vegetables, green leafy vegetables, fruits, meat, eggs, milk, curd and buttermilk and fish, Dhal, bread, millet, snack, "wheat kalli" (pasta dish made from wheat flour and water), Boost (powdered drink) and food, in general, have also been mentioned by more than a woman. But the interesting fact is that half of the women changed their eating habits during pregnancy by excluding certain foods. The reasons given by most women were vomiting and food products classified as "gas production". These foods were cabbage, dhal, potatoes, beans and fatty foods. One woman mentioned that she was avoiding buttermilk, glucose and soft coconut because these foods would make the baby fat, which would make childbirth difficult. Very also mentioned the exclusion of papaya because of its effect of "warming" on the mother and the baby.

If we speak of the qualitative study of nutrition beliefs and practices during pregnancy and breastfeeding in a rural area of Karnataka in India, it also shows that the results of these practices clearly indicate the predominance of the powerful influence of culture and culture. tradition in this field. The results concerning the practices clearly indicate the predominance of the powerful influence of culture and traditions in this field. Many of the beliefs stated for each food had no scientific explanation. Common and inexpensive vegetables such as pumpkin and cucumber were limited and ghee and ragi were not acceptable during pregnancy. The belief in apple cream was interesting because it was avoided because it caused the cold. Beliefs and restrictions can have a negative impact on pregnant and lactating women, generation after generation [18]. 
The latest study by Banu et al. investigated existing beliefs and practices regarding feeding during pregnancy and breastfeeding as part of a cross-sectional / community-based experimental study of 650 pregnant or lactating women in different regions of Tamilnadu. The study showed that the highest percentage ( $89 \%$ ) of women thinking that papaya, ripe papaya, pineapple, fish, yams, eggs and raw poultry meat were hot foods and that consumption of these foods during pregnancy caused abdominal pain and uterine contractions. A majority (93\%) of women thought that curds, butter, cheese, amla (grapes), grapes, green leafy green apple vegetables are considered cold and that consumption of these foods during pregnancy causes a cold and fever in the mother and during breastfeeding causes colds and coughs in children. The study found that there are food taboos and misconceptions about pregnancy in the state of Tamilnadu. Women in this study area were required to avoid certain foodstuffs because of their cultural and traditional views [19]. All the above study showed that there is a high impact of customs, believes, taboos etc. on the section on food items. The subsequent study by Payghan et al was conducted to determine existing practices, knowledge and attitudes of mothers regarding nutrition during pregnancy in urban and rural areas. This crosssectional study was conducted among pregnant women visiting the antenatal clinic in the urban and rural field practice area from June to November 2012. The knowledge and nutritional practices of urban mothers are high compared to rural mothers, while rural and urban mothers are positive attitude towards nutrition. It has been shown that $68 \%$ of urban respondents and $73 \%$ of rural respondents avoid certain foods such as papaya, coconut and meat during pregnancy. Urban mothers $(67 \%)$ were more aware of their diet than rural mothers $(50 \%)$ [20].

\subsection{Socio-Economic Characteristics}

The nutritional behaviour of the pregnant and lactating women also affected by the socio-economic condition of the women. Knowledge \& Awareness, Family Income, and Socioeconomic is the most contributing factor to affecting the nutritional behaviour of a woman. Many studies have been done which have stated the positive and negative aspects of the socio-economic factors regarding Dietary intake.

The study found that median intake of energy and protein were higher in pregnant women belonging to be lower socioeconomic class and lower in upper/ middle socioeconomic class. The study also stated that no statically significant differences were found in the intake of energy and protein by non-pregnant women of the same class. This study also highlighted that energy and protein intakes were found higher in pregnant women those belonging to joint family and lower in the nuclear family [12].

The study also indicated that low caloric intake in pregnant women and non-pregnant women may be due to insufficient knowledge of food intake and socio-economic status. This study also shows that lack of knowledge about the amount of food intake to be taken. Caloric intake in both pregnant and non-pregnant women may be due to low socio-economic status, with most subjects of higher, lower, middle socioeconomic status. The author also stated that insufficient knowledge of appropriate dietary intake may be another possible explanation [12].

Family income studies have shown a significant impact on dietary intake. Family income is also a factor influencing the feeding behaviour of pregnant and lactating women. The study in northern India has selected 90 pregnant women living in rural areas in both agricultural and non-agricultural 
communities. The authors found in this study that the consumption of green leafy fruits and vegetables had a positive relationship with the family's monthly income. The increase in green fruit and vegetable consumption has been accompanied by an increase in the family's monthly income. The consumption of green leafy fruits and vegetables has increased with the increase in the family's monthly income. There is an urgent need to educate pregnant women in rural areas about their increased nutritional needs [21].

The period of pregnancy and breastfeeding of the mother is a crucial period where nutritional knowledge is important to influence the mother's eating habits. The study, which reported that changes after intervention in knowledge, attitude and practices were $47 \%, 22 \%$ and $16 \%$ respectively. After the intervention, it was observed that only $18 \%$ of respondents had changed their nutritional intake during pregnancy and breastfeeding. According to the study, after the intervention of a program, consumption of a healthy diet was taken by only $16 \%$ of respondents. A large number of respondents did not want to improve their food consumption. This may have been due to the fact that the diet was categorically traditional for reasons of food intake or poverty [22].

\section{Conclusion}

The feeding practices, dietary intakes and nutritional status of pregnant and lactating women were below national and international recommendations. Therefore, continuing education on health and nutrition is recommended for women, their families, and their communities regarding increased dietary intake, appropriate dietary practices, and dietary diversification during breastfeeding and pregnancy to improve the health and nutrition outcomes of breastfeeding and pregnant women [23]. The main warning nutrients in the diet of mothers were energy, protein, vitamin $A$ and calcium. In 80 percent of women, no extra food is taken during pregnancy and lactation, which in turn reflects the nutritional status of newly born [2].

Thus, there is a strong need to provide nutrition education to the mothers that the dietary requirement of pregnant and lactating mothers is more than non-pregnant [2]. More research and intervention are required on pregnant and lactating women.

\section{Acknowledgements}

I would like to extend my gratitude to all those people who encouraged me to complete this paper. I would also like to express my gratitude towards my family for constant encouragement and inspiration throughout my work. This study has no fund from any sponsoring agency.

\section{References}

[1] Udipi, S., Ghugre, P., \& Antony, Under Nutrition in Pregnancy and Lactation. J Indian Med Assoc, 111(9),2002, 548-57. https://doi.org/10.1159/000362299

[2] ICMR. Nutrient Requirements and Recommended Dietary Allowances for Indians a. Indian Council of Medical Research, 1-334,2009.

[3] Pathak, P., Kapil, U., Kapoor, S. K., Saxena, R., Kumar, A., Gupta, N., ... Singh, P. (2004). Prevalence of multiple micronutrient deficiencies amongst pregnant women in a rural area of Haryana. Indian Journal of Pediatrics, 71(11),2004, 1007-14. https://doi.org/10.1007/BF02828117 
[4] Singh, M. B., Fotedar, R., \& Lakshminarayana, J. (2009). Micronutrient deficiency status among women of desert areas of western Rajasthan, India. Public Health Nutr, 12(5), 624-629. https://doi.org/S1368980008002395 [pii]ไr10.1017/S1368980008002395 [doi]

[5] Bhatia, B. D., Banerjee, D., Agarwal, D. K., \& Agarwal, K. N. Dietary intakes of urban and rural pregnant, lactating and non-pregnant, non-lactating vegetarian women of Varanasi. Indian Journal of Medical Research, 74(5),1981, 680-687. Retrieved from http://www.scopus.com/inward/record.url?eid=2-s2.00019814694\&partnerID=40\&md5=f409d396c09853b24e3118b5f2075b04

[6] Asha, K., \& Salil, S.Nutrient Intake of Lactating Mothers from Rural areas and urban areas. Indian Journal of Social Research, 39(April-June), 1998, 2.

[7] Saxena, N. C. Hunger, Under-nutrition and food security in India. Chronic Poverty Research Center, 5-7,2008. Retrieved from http://mercury.ethz.ch/serviceengine/Files/ISN/128320/ipublicationdocument_singledocument/cb f04f5c-7669-4815-904c-c21364eddd8b/en/CPRC-IIPA+44.pdf

[8] Jood, S., Bishnoi, S., \& Khetarpaul, N. Nutritional Status of Rural Pregnant Women. Indian Journal of Community Medicine, 16(3),2002, 121-131.

[9] Agarwal, K. N., Agarwal, D. K., Sharma, a Sharma, K., Prasad, K., Kalita, M. C., Kumari, P. Prevalence of anaemia in pregnant \& lactating women in India. The Indian Journal of Medical Research, 124(2), 2006, 173-184. https://doi.org/10.3906/sag-0811-44

[10] Andersen, L. T., Thilsted, S. H., Nielsen, B. B., \& Rangasamy, S. Food and nutrient intakes among pregnant women in rural Tamil Nadu, South India. Public Health Nutrition, 6(2), 2003,131-7. https://doi.org/10.1079/PHN2002367

[11] Bairwa, K., Lakhawat, S., Bairwa, T., \& Verma, A. K. An Exploratory Study of Diet and Nutritional Status of Shariya Tribe Lactating Women in Baran District of Rajasthan. International Journal of Science, Environment and Technology, 6(1),2017, 927-934.

[12] Kumar, R., Aslesh, O. P., Anjali, Kapoor, A., Sanan, D., Wadhwa, E., \& Agarwal, N. Urban poor women do not increase their diet during pregnancy: A study from an urban resettlement colony in Delhi, India. International Journal of Medicine and Public Health, 3(3),2013, 134. https://doi.org/10.4103/2230-8598.118944

[13] Ghosh-Jerath, S., Devasenapathy, N., Singh, A., Shankar, A., \& Zodpey, S. Ante natal care (ANC) utilization, dietary practices and nutritional outcomes in pregnant and recently delivered women in urban slums of Delhi, India: an exploratory cross-sectional study. Reproductive Health, 12(1), 2015, 20. https://doi.org/10.1186/s12978-015-0008-9

[14] Kajale, N. A., Khadilkar, V. V., Mughal, Z., Chiplonkar, S. A., \& Khadilkar, A. V. Changes in body composition of Indian lactating women: a longitudinal study. Asia Pacific Journal of Clinical Nutrition, 25(3),2016, 556-562. https://doi.org/10.6133/apjcn.092015.16

[15] Sinha, R., Sharma, B., \& Mahanta, P. Intake of nutrients and its food sources among selected tribal expectant mothers of Ranchi district of Jharkhand. Journal of Dairying, Foods and Home Sciences, 26(2),2007, 122-127. Retrieved from http://arccjournals.com/pdf/Dairying/jdfhs-26-2/jdfhs-26-2012.pdf\%5Cnhttp://www.arccjournals.com/pdf/Dairying/jdfhs-26-2/jdfhs-26-2-012.pdf

[16] Agrahar-Murugkar, D., \& Pal, P. P. Intake of nutrients and food sources of nutrients among the Khasi tribal women of India. Nutrition, 20(3),2004, 268-273. https://doi.org/10.1016/j.nut.2003.11.008

[17] Joshi, S., \& Singh, V. Assessment of food related habits and customs of Bhil tribe of Udaipur district, Rajasthan. Food Science Research Journal, 6(2), 2015, 333-340. https://doi.org/10.15740/HAS/FSRJ/6.2/333-340

[18] Catherin, N., Rock B, Roger V, Ankita C, Ashish G, Delwin P, Deeepthi S, Goud BR. Beliefs and practices regarding nutrition during pregnancy and lactation in a rural area in Karnataka, India: a qualitative study. International Journal of Community Medicine and Public Health, 2(2),2015, 116. https://doi.org/10.5455/2394-6040.ijcmph20150509 
[19] Banu, K., A, P., B, A., \& Ismail, M. Neonatal outcome in meconium stained amniotic fluid-oneyear experience. International Journal of Biomedical and Advance Research, 7(8), 2016,393-396. https://doi.org/10.7439/ijbar

[20] Payghan, B., Kadam, S. S., \& Reddy, R. M. Research \&amp; reviews: journal of medical and health sciences. Research \& Reviews: Journal of Medical and Health Sciences, 3(4), 2012, 95-99. Retrieved from https://www.rroij.com/open-access/a-comparative-study-of-nutritional-awarenessamong-urbanrural-pregnant-mothers.php?aid=35014

[21] Panwar, B., \& Punia, D. Nutrient intake of rural pregnant women of Haryana state, northern India: relationship between income and education. International Journal of Food Sciences and Nutrition, 49(5),2009, 391-395.Retrieved from http://ovidsp.ovid.com/ovidweb.cgi?T=JS\&PAGE=reference $\& D=$ med4\&NEWS=N\&AN=10367 009

[22] Bhagyapreet, K., \& Himanshu, D. Nutritional Status of Pregnant Women and Lactating Mothers and An Intervention of "Kap" of Dietary Practices among Dangolion Tharu Schedule Tribes Womens. Research Journal of Family Res. J. Family, 2(6),2014, 6-9.

[23] Ministry of Health. Food and nutrition guidelines for healthy adults: A background paper,2006. https://doi.org/ISBN 978-0-478-19380-0

\footnotetext{
*Corresponding author.

E-mail address: dhakad30@ gmail.com
} 